F $\quad$ Faculty

A Aid to

A CPD Self-Assessment Test

C CPD

T Topics

QUESTION SHEET

Review No. 2001/03

To be reviewed not later than 31 May 2006

\title{
Resuscitation in the family planning and reproductive health care setting
}

Indicate your answer by ticking the appropriate box for each question

1. Cervical stimulation produces an anaphylactic reaction.

2. Bradycardia is defined as a ventricular rate below $60 \mathrm{~b} / \mathrm{min}$.

3. Atropine is the drug of choice for reversing bradycardia.

4. Signs of 'cervical shock' include pallor, sweating, and tachycardia.

5. If 'cervical shock' occurs, the first line of action is to raise the legs and lower the head.

6. Basic life support should commence immediately after cessation of breathing.

7. HIV has been transmitted to person giving rescue breathing.

8. Survival is rare if defibrillation and/or drug therapy is unavailable within 30 minutes of cardiac arrest.

True

False

9. IV adrenaline is the drug of choice for anaphylaxis.

10. Current adult basic life support guidelines recommends a compression to ventilation ratio of 15:2.

Turn to page 173 for answers 